\title{
Photoacoustic Investigation of Multilayer Semiconductor Materials
}

\author{
Yao-chun Shen ${ }^{1}$ and Shu-yi Zhang ${ }^{1}$
}

Received March 23, 1993; revised March 1, 1994

\begin{abstract}
A one-dimensional model to investigate the photoacoustic (PA) generation and depth profiling in multilayer semiconductor materials has been developed by considering the contributions of thermal and plasma waves. The theoretical results show that the contribution of the plasma wave induced by photo-generated carriers (PGC) to the photoacoustic signal through the electro-elastic effect is predominant at high modulation frequencies and the contribution of the thermal wave, which is caused by the recombination of PGC, to PA signal through the thermo-elastic effect is more important at low modulation frequencies. We also investigated the depth profiling ability of PA detection by changing either the modulation frequency or the phase shift of the reference signal. The theoretical analyses are consistent with the experimental results.
\end{abstract}

KEY WORDS: Photoacoustic; thermal wave; plasma wave; acoustic wave; depth characterization.

\section{INTRODUCTION}

In the past decade, the photoacoustic (PA) technique has been successfully applied to study semiconductor materials and devices.(1) One of the most promising applications of the technique is the laminated characterization of layered materials.(2) Opsal and Ronsencwaig $^{(3)}$ developed a one-dimensional (1-D) model (O-R theory) of thermal wave depth profiling in multilayer materials, in which they considered a semiinfinite layered sample with discontinuous thermal impedance between two neighboring layers and obtained expressions for the temperature at the surface and the elastic response beneath the surface of the sample. On the basis of O-R theory, we have investigated the mechanism of depth profiling in piezoelectric PA detection for a multilayered sample, under thermally thick but acoustically thin conditions, in theory and experiment. ${ }^{(4)}$ In the theories above, the authors considered that the PA generation is predominantly due to the thermoelastic ef-

\footnotetext{
${ }^{1}$ Institute of Acoustics and Lab of Modern Acoustics, Nanjing Uni-
} versity, Nanjing 210008 , China. fect. In fact, due to the diffusion and recombination of photo-generated carriers (PGC) in semiconductors, there are significant changes in the distribution of thermal and plasma wave sources, and moreover, the electro-elastic strain contribution to the PA generation cannot be neglected. ${ }^{(5)}$ In this paper, we present experimental and theoretical investigations of the PA generation and depth profiling in multilayer semi-conductor materials. In the experiment, the PA signal of a Si wafer containing Sbdoped areas is measured as a function of modulation frequency. The experimental results show that some discrepancy exists in comparing with previous theories, considering only the thermoelastic effect. We present here a 1-D multilayer theory in which both thermoelastic and electro-elastic effects have been taken into account. The PA signals caused by the elastic sources (thermal and plasma waves) at arbitrary depth have been calculated, using a Green's function method. In addition, the influences of thermal and electronic properties of the subsurface layer on the surface temperature and PA signal have also been calculated. Theoretical results are consistent with those of experiment. 


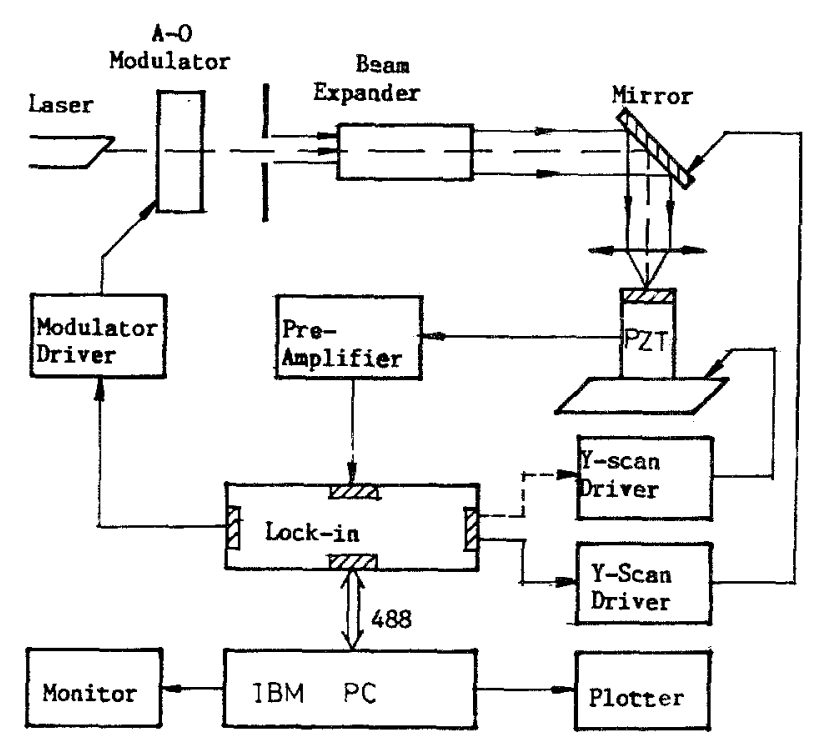

Fig. 1. Schematic diagram of the PA detection system.

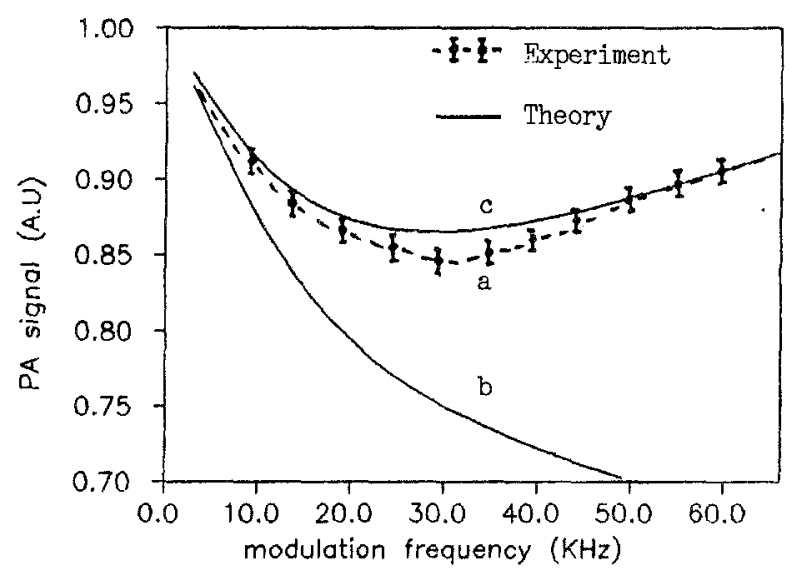

Fig. 2. Normalized PA signal vs. modulation frequency: (a) experimental results, (b) theoretical results, when only thermoelastic strain is considered, (c) theoretical results, when both thermoelastic and electro-elastic strains are considered.

\section{EXPERIMENT}

Figure 1 shows a schematic diagram of the PA detection system. A modulated laser beam is incident on a sample, which is a locally Sb-doped $\mathrm{Si}$ wafer, covered by an epitaxial layer of Si. The PA signal detected by the PZT transducer is amplified by a lock-in amplifier and then sent to a computer. Using this experimental system, we have obtained PA laminated images of the sample. ${ }^{(4)}$ Next, in order to eliminate the effect of the frequency response of the system, the PA signals of a Si substrate and the buried Si substrate are measured separately as a function of modulation frequency. The

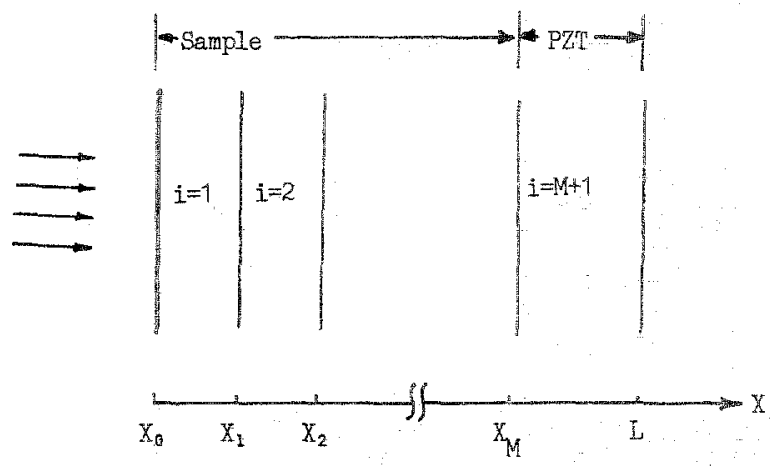

Fig. 3. 1-D theoretical model of multilayered naterials.

normalized PA signal, i.e., the ratio of the PA signals obtained from the latter to the former, vs. modulation frequency is shown in Fig. 2 (dashed line). From the curve we can see that there is a "concavity" at a frequency of about $30 \mathrm{KHz}$, which will be discussed in the following sections.

\section{THEORY}

A plane laser beam with modulation frequency, $\omega$, is incident on the surface of a multilayer semiconductor sample (Fig. 3). As the photon energy ho is greater than the band gap energy $E_{g}$ of the semiconductor, both ther$\mathrm{mal}$ and plasma waves are excited. For simplicity, we assume that: (i) the thickness of the sample is greater than the plasma and thermal wavelengths, and (ii) the sample is uniform in the transverse direction and multilayered in depth. Thus, a 1-D model can be used.

\subsection{Plasma Wave}

The recombination and diffusion of $\mathrm{PGC}$ in the ith layer ( $i=1$ to $M$ ) of the sample can be described by the 1-D wave equation

$$
\frac{d^{2} N_{i}(x)}{d x^{2}}-p_{i}^{2} N_{i}(x)=-\frac{\beta_{i}}{h v D_{i}} I_{i}(x)
$$

where $I_{i}(x)$ is the laser intensity, $N_{i}(x)$ is the density of PGC, and $p_{i}$ is the wave vector of the plasma wave, defined as

$$
p_{i}^{2}=\frac{1+j \omega \tau_{i}}{D_{i} \tau_{i}}
$$

and $\beta_{i}, D_{i}, \tau_{i}$ are the optical absorption coefficient, the ambipolar diffusion coefficient and the lifetime of PGC, respectively. 
According to the continuity of the density and flux of PGC, as well as the recombination of PGC at the surface and interface of the sample, the boundary conditions are

$$
\begin{array}{ll}
N_{i}\left(x_{i}\right)=N_{i+1}\left(x_{i}\right) & \\
D_{i+1} \frac{d N_{i+1}(x)}{d x}-D_{i} \frac{d N_{(}(x)}{d x}=-S_{i+1} N_{i+1}(x) & \left(x=x_{i}\right) \\
D_{1} \frac{d N_{1}(x)}{d x}=S_{1} N_{1}(x) & (x=0) \\
N_{M}\left(x_{M}\right)=0 &
\end{array}
$$

where $S_{i}$ is the surface or interface recombination rate of PGC. The solution of Eq. (1) can be written as

$$
N_{i}(x)=A_{i} e^{-p_{i} x}+B_{i} e^{-p_{i} x}+f_{1 i}(x)
$$

The coefficients $A_{v} B_{i}$ can be determined by the boundary conditions (Eq. 3), and the particular solution, $f_{1 i}(x)$, obtained directly from Eq. (1), is given in the Appendix.

\subsection{Thermal Wave}

The 1-D thermal wave equation in the $i$ th $(i=1$ to $M$ ) layer of the sample can be written as:

$$
\frac{d^{2} T(x)}{d x^{2}}-q_{i}^{2} T_{i}(x)=-\frac{E_{g}}{\kappa_{i} \tau_{i}} N_{i}(x)-\frac{h v-E_{g}}{h v K_{i}} \beta_{i} I_{i}(x)
$$

where $T_{i}(x)$ is the temperature and $q_{i}$ is the thermal wave vector, defined as:

$$
q_{i}^{2}=\frac{j \omega p_{i} c_{i}}{\kappa_{i}}
$$

where $\rho_{i}, c_{i}, \kappa_{i}$ are the density, the specific heat, and the thermal conductivity, respectively. The source of the periodic heating in the semiconductor includes two parts: the first term on the right hand side of Eq. (5) results from the bulk recombination of the PGC, and the second term comes from the rapid de-excitation of PGC to the bottom of the conduction band. Considering the continuity of the temperature and heat flux, as well as the recombination of $\mathrm{PGC}$ at the surface and interface of the sample, the boundary conditions are

$$
\begin{array}{ll}
T_{i}\left(x_{i}\right)=T_{i+1}\left(x_{i}\right) & \\
\kappa_{i+1} \frac{d T_{i+1}(x)}{d x}-\kappa_{i} \frac{d T_{i}(x)}{d x}=-S_{i+1} N_{i+1}(x) E_{g} & \left(x=x_{i}\right) \\
\kappa_{1} \frac{d T_{1}(x)}{d x}=-S_{1} N_{1}(x) E_{g} & (x=0) \\
T_{M}\left(x_{M}\right)=0 &
\end{array}
$$

The solution of Eq. (6) can be written as

$$
T_{i}(x)=E_{i} e^{-q_{i} x}+F_{i} e^{-q_{i} x}+f_{2 i}(x)
$$

The coefficients $E_{i}$ and $F_{i}$ can be determined by the boundary conditions (Eq. 7), and the particular solution, $f_{2 i}(x)$, can be obtained directly from Eq. (5), as shown in the Appendix.

\subsection{Elastic Response}

As discussed by Kino et al., ${ }^{(5)}$ the elastic strain in semiconductor materials includes two parts. The first is the thermoelastic strain that results from the periodic heating associated with the thermal wave, and the second is the electro-elastic strain that is due to the injection of PGC associated with plasma wave. Thus, the 1-D elastic wave equation in the $i$ th $(i=1$ to $M$ ) layer of the sample or in the PZT transducer $(i=M+1)$ is

$$
\frac{d^{2} \phi_{i}(x)}{d x^{2}}+k_{i}^{2} \phi_{i}(x)=\beta_{t i} T_{i}(x)+\beta_{e i} N_{i}(x)
$$

and

$$
\begin{aligned}
& \beta_{t i}=\frac{2 \mu_{i}+3 \lambda_{i}}{2 \mu_{i}+\lambda_{i}} \alpha_{t i} \\
& \beta_{e i}=\frac{2 \mu_{i}+3 \lambda_{i}}{2 \mu_{i}+\lambda_{i}} \alpha_{e i}
\end{aligned}
$$

where $\phi_{i}(x)$ is the elastic displacement potential, $\lambda_{i}$ and $\mu_{i}$ are the Lame constants, $k_{i}$ is the acoustic wave vector, and $\alpha_{i t}, \alpha_{e i}$ are the thermal expansion coefficient and the electro-elastic strain constant of the $i$ th layer of the sample, respectively. From the elastic displacement potential, $\phi_{i}(x)$, we can find the displacement $U_{i}(x)$ and stress $\sigma_{i}(x)$

$$
\begin{gathered}
U_{i}(x)=\frac{d \phi_{i}(x)}{d x} \\
\sigma_{i}(x)=-\rho_{i} \omega^{2} \phi_{i}(x)
\end{gathered}
$$

Since the surface of the sample and the bottom of the PZT transducer are free, and the stress and displacement are continuous at the interface of the sample, the boundary conditions are:

$$
\begin{aligned}
& \sigma_{1}(0)=0 \\
& \sigma_{M+1}(L)=0 \\
& \sigma_{i}\left(x_{i}\right)=\sigma_{i+1}\left(x_{i}\right) \\
& U_{i}\left(x_{i}\right)=U_{i+1}\left(x_{i}\right)
\end{aligned}
$$

The solution of Eq. (6) with the boundary conditions above is

$$
\phi_{i}(x)=G_{i} e^{-j k i x}+H_{i} e^{i k_{i} x}+f_{3 i}(x)
$$

The coefficients $G_{i}$ and $H_{i}$ can be determined by the boundary conditions (Eq. 13), and the particular solu- 
tion, $f_{3 i}(x)$, obtained directly from Eq. (9), is provided in the Appendix. According to the piezoelectric equation, the detected PA signal, i.e., the output voltage of the PZT transducer is

$$
V=h_{33} k_{M+1}^{2} \int_{x_{M}}^{L} \phi_{M+1}(x) d x
$$

where $h_{33}$ is the piezoelectric constant of the PZT.

\subsection{Depth Characterization}

The PA signal given in Eq. (15) is the total contribution of acoustic sources in the different depths of the sample. In order to investigate the laminated detection ability of the PA technique, we should obtain the output voltage of the PZT produced by a source at a depth $x$ beneath the surface. Since the acoustic wavelength is much larger than the thickness of the sample, the difference of the elastic features in the sample is neglected, and the solution of Eq. (9) can be expressed by the integral of the Green"s function

$$
\phi(x)=\int_{0}^{x_{M}} G\left(x, x^{\prime}\right) f\left(x^{\prime}\right) d x^{\prime}
$$

where

$$
f\left(x^{\prime}\right)=\beta_{t i} T_{i}\left(x^{\prime}\right)+\beta_{e i} N_{i}\left(x^{\prime}\right)
$$

and the Green's function, $G\left(x, x^{\prime}\right)$, describes the elastic response at $x$ due to a $\delta$ function source at $x^{\prime}$, and satisfies following equation

$$
\frac{\partial^{2} G\left(x, x^{\prime}\right)}{\partial x^{2}}+k_{i}^{2} G\left(x, x^{\prime}\right)=\delta\left(x, x^{\prime}\right)
$$

The solution to Eq. (18) can be obtained ${ }^{(4)}$ as

$$
G\left(x, x^{\prime}\right)=\frac{2}{L} \sum_{n=1}^{\infty} \frac{\sin \left(a_{n} x\right)}{k_{i}^{2}-a_{n}^{2}} \sin \left(a_{n} x^{\prime}\right)
$$

where $a_{n}=n \lambda / L$. Thus, the output voltage of the PZT produced by the acoustic source at $x^{\prime}$ is

$$
V\left(x^{\prime}\right)=f\left(x^{\prime}\right) \sum_{n=1}^{\infty} C_{n} \sin \left(a_{n} x^{\prime}\right)
$$

and the constant $C_{n}$ is determined only by the parameter of the PZT transducer, namely:

$$
C_{n}=\frac{2 h_{33} k_{M+1}^{2}}{\left(k_{M+1}^{2}-a_{n}^{2}\right) L} \int_{x_{M}}^{L} \sin \left(a_{n} x\right) d x
$$

If the PZT is operated near its resonant frequency, only the $n$th term in the summation of Eq. (20) should be considered. Moreover, the plasma and thermal wavelengths are much smaller than the thickness of the sample. Thus, $a_{n} x^{\prime}<<1$ and $\sin \left(a_{n} x^{\prime}\right)$ approaches the value $a_{n} x^{\prime}$. As $n$ is not very large, a good approximation of Eq. (20) is

$$
V\left(x^{\prime}\right)=C_{n} a_{m} x^{\prime} f\left(x^{\prime}\right)
$$

Generally, the PA signal is mixed with a reference signal, using a lock-in amplifier, to improve the signalto-noise ratio. Then the imaging signal is

$$
V_{0}\left(x^{\prime}\right)=\mathrm{R}\left[V\left(x^{\prime}\right) e^{j \psi_{0}}\right]=\left|V\left(x^{\prime}\right)\right| \cos \left[\psi\left(x^{\prime}\right)-\Psi_{0}\right]
$$

where $\Psi_{0}$ is the phase shift of the reference signal and $\psi\left(x^{\prime}\right)$ is the phase lag of the PA signal caused by the source at depth $x^{\prime}$, and which contains the information on the thermal and electronic features of the material at a depth $x$ '. Since the structures at different depths induce different phase lags, the PA signals resulting from the different subsurface features can be strengthened selectively by adjusting the phase shift of the reference signal. On the other hand, the total PA signal is the integral of the PA signals produced by the sources at different depths, that is

$$
\begin{aligned}
\bar{V} & =\int_{0}^{x_{M}} V_{0}\left(x^{\prime}\right) d x^{\prime} \\
& =\int_{0}^{x_{a}} V_{0}\left(x^{\prime}\right) d x^{\prime}
\end{aligned}
$$

where $x_{M}$ is the thickness of the sample, and $x_{a}$ is the effective integral region, i.e., the penetration depth of the thermal and plasma waves. On the other hand, due to the fact that the plasma and thermal wavelengths vary inversely with the square root of the modulation frequency, subsurface features at different depths can also be obtained by changing the modulation frequency. (6)

\section{THE NUMERICAL RESULTS AND DISCUSSIONS}

As an example, the buried Si wafer with three layers measured in the experiment is used for the calculation. The thickness of the epitaxial layer and doped layer about $10 \mu \mathrm{m}$ and $3 \mu \mathrm{m}$, respectively. The numerical results are as follows: 


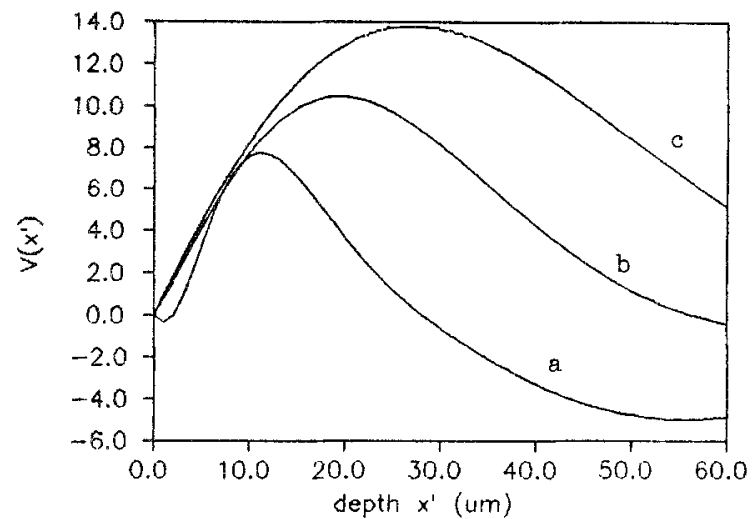

Fig. 4. Contribution of source at depth $x^{\prime}$ to the PA signal changes with depth $x^{\prime}$, at moduiation frequencies of: (a) $300 \mathrm{KHz}$, (b) $100 \mathrm{KHz}$, (c) $40 \mathrm{KHz}$.

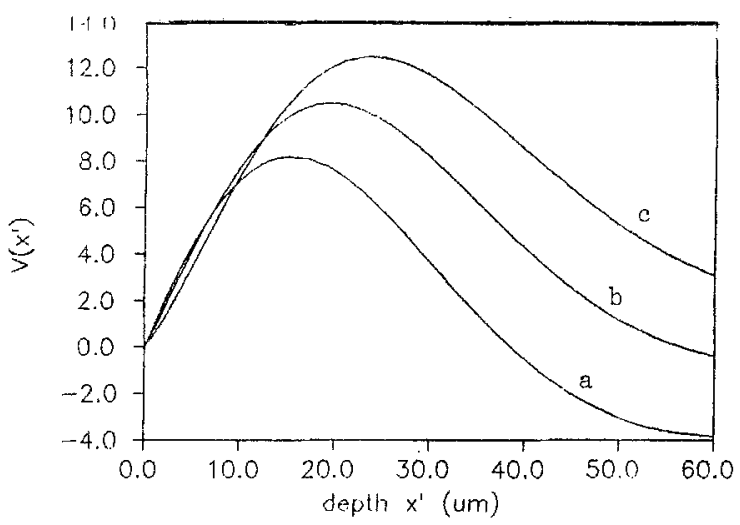

Fig. 5. Contribution of source at depth $x^{\prime}$ to the PA signal changes with depth $x^{\prime}$, at a modulation frequency of $100 \mathrm{KHz}$. The phase shifts of the reference signal are: (a) $100^{\circ}$, (b) $130^{\circ}$, (c) $150^{\circ}$.

tained from different frequencies. As the modulation frequency decreases from $300 \mathrm{KHz}$ to $40 \mathrm{KHz}$, the depth of the source, corresponding to the maximum contribution to the PA signal, changes from about $12 \mu \mathrm{m}$ to 28 $\mu \mathrm{m}$ due to the increase of the plasma and thermal wavelengths. On the other hand, from Fig. 5, in which the different curves represent different phase shifts of the reference signal, we can see that, as the phase shift increases from $110^{\circ}$ to $150^{\circ}$, the depth of the source, corresponding to the maximum contribution to the PA signal, changes from about $15 \mu \mathrm{m}$ to $25 \mu \mathrm{m}$. We can conclude from the curves that the contribution of the different layers of the sample can be suppressed or strengthened by adjusting the modulation frequency or the phase shift of the reference signal. Thus, the important laminated characterization ability of the PA technique is provided by either adjusting the phase shift or

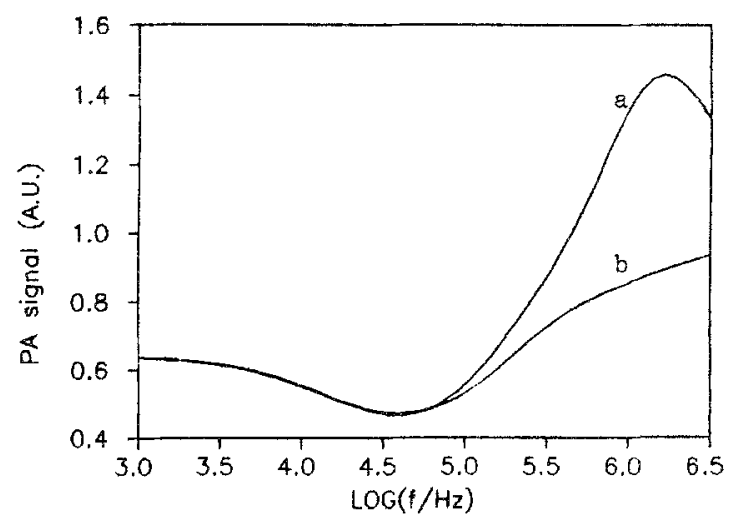

Fig. 6. Normalized PA signal vs. modulation frequency. The electroelastic effect is not considered in (a) and is considered in (b).

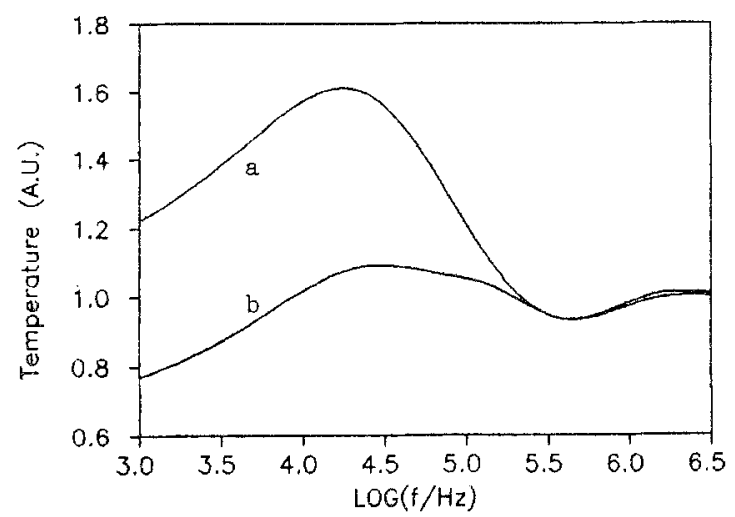

Fig. 7. Normalized surface temperature versus modulation frequency. The influence of PGC is not considered in (a) and is considered in (b).

by changing the modulation frequency. These results are in agreement with those of our experiments. ${ }^{(4,6)}$ In general, the former is much easier in practice than the latter.

\subsection{The Contribution of PGC}

The influences of PGC on the surface temperature and the PA signal for different modulation frequencies are demonstrated in Figs. 6 and 7. The curves have been normalized by the PA signal from the Si substrate in order to eliminate the effect of the frequency response. From the curves in Fig. 6 we can see that the influence of PGC on PA signal through the electro-elastic effect is important at high modulation frequencies, but it can be neglected at low frequencies. From Fig. 7 we can see that the contribution of PGC to the surface temperature is important at low modulation frequencies, while it is negligible at high modulation frequencies due to the rapid decay of plasma and thermal waves in depth. It 


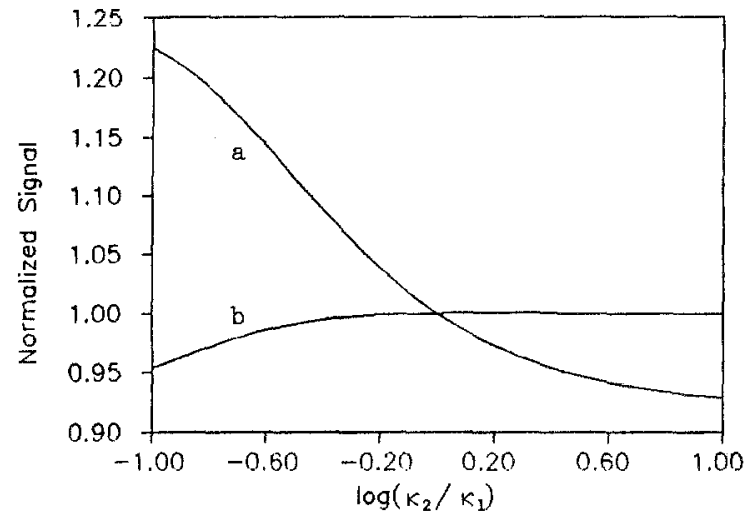

Fig. 8. Normalized surface temperature (a), and PA signal (b) vs. thermal conductivity at a modulation frequency of $100 \mathrm{KHz}$.

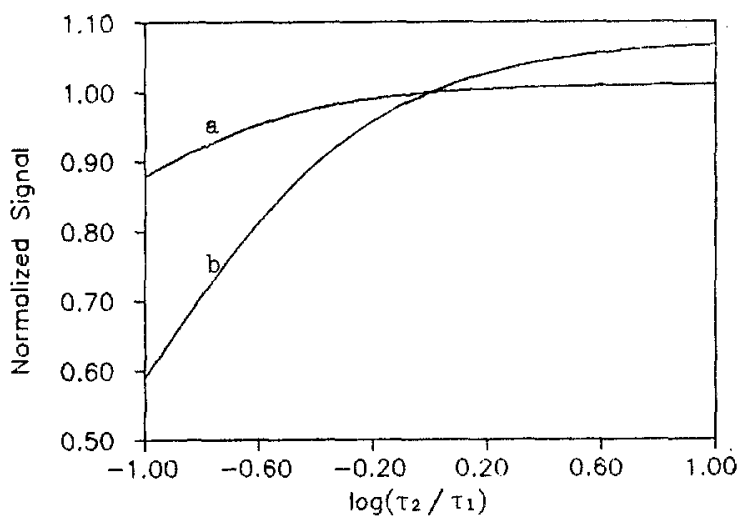

Fig. 9. Normalized surface temperature (a), and PA signal (b) vs. $\mathrm{PGC}$ lifetime at a modulation frequency of $100 \mathrm{KHz}$.

should be pointed out that the influence of PGC on PA signal at low modulation frequencies cannot be neglected, since the thermal wave caused by the recombination of PGC can also produce a PA signal through the thermoelastic effect.

In order to compare with experimental results, the PA signal vs. modulation frequency is calculated with proper parameters and is shown in Fig. 2. The calculated PA signals have also been normalized. The curve (b) is the PA signal calculated by considering only thermoelastic strain. The curve (c) is the theoretical result, considering both thermoelastic and electro-elastic strains, and is consistent with the experimental result shown in curve (a). The appearance of the "concavity" can be explained by superimposing both contributions. We can conclude that the plasma wave has an important, and even dominant, contribution to the PA generation in semiconductors at relatively high modulation frequencies.

\subsection{Influence of Subsurface Features}

Figures 8 and 9 illustrate the effect of the parameters of the buried layer on the surface temperature and PA signal. The signals are all normalized by the $\mathrm{Si}$ sample for the same reason as described above. From the curves we can see that the surface temperature is more sensitive to thermal conductivity than the PA signal, while the PA signal is more sensitive to the electronic property (PGC lifetime). The curves also show that the surface temperature or PA signal has high sensitivity for $\kappa_{2}$ or $\tau_{2}$, respectively, when the parameters $\left(\kappa_{2}, \tau_{2}\right)$ of the buried layer are smaller than those of $\mathrm{Si}$ wafer. Fortunately, the condition is satisfied for most semiconductor materials. Therefore, high sensitivity can be obtained by the PA technique.

\section{CONCLUSION}

From the discussions above we can conclude as follows: (i) The contribution of the subsurface structures at different depths to the PA signal can be weakened of strengthened by changing either the modulation frequency or the phase shift of the reference signal. However, their mechanisms are different: the former is due to the changes of the plasma and thermal wavelengths, while the latter is due to the changes of the contribution of the acoustic sources (plasma and thermal waves) at depth $x^{\prime}$. (ii) The influence of PGC on PA signal is important at both low and high modulation frequencies. At high modulation frequency, PGC generates a PA signal through the electro-elastic effect. At low modulation frequency, the recombination of PGC results in a thermal wave, which then generates the PA signal through thermoelastic effect. (iii) The surface temperature is more sensitive to thermal conductivity than the PA signal, while the PA signal is more sensitive to the electronic property (PGC lifetime). However, the surface temperature or PA signal has high sensitivity for $\kappa_{2}$ or $\tau_{2}$, re. spectively, when the parameters $\left(\kappa_{2}, \pi_{2}\right)$ of the buried layer are smaller than those of Si wafer.

\section{ACKNOWLEDGMENT}

This work is supported by Natural Science Foundation of China, Grant No. 19134040. 
APPENDIX

$$
\begin{aligned}
& f_{1 i}(x)=-\frac{\beta_{i} I_{i}(x)}{h v D_{i}\left(\beta_{i}^{2}-p_{i}^{2}\right)} \\
& f_{2 i}(x)=-\frac{E_{g}}{\kappa_{i} \tau_{i}\left(p_{i}^{2}-q_{i}^{2}\right)}\left[A_{i} e^{-p_{i} x}+B_{i} e^{p_{i} x}\right] \\
& -\frac{D_{i} \tau_{i}\left(\beta_{i}^{2}-p_{i}^{2}\right)\left(h v-E_{g}\right)-E_{g}}{h v \kappa_{i} D_{i} \tau_{i}\left(\beta_{i}^{2}-p_{i}^{2}\right)\left(\beta_{i}^{2}-q_{i}^{2}\right)} \beta_{i} I_{i}(x) \\
& f_{3 i}(x)=\beta_{i i}\left[\frac{E_{i} e^{-q_{i} x}+F_{i} e^{q_{i} x}}{q_{i}^{2}+k_{i}^{2}}-\frac{E_{g}}{\kappa_{i} \tau_{i}} \frac{A_{i} e^{-p_{i} x}+B_{i} e^{p_{i} x}}{\left(p_{i}^{2}+k_{i}^{2}\right)\left(p_{i}^{2}-q_{i}^{2}\right)}\right. \\
& \left.-\frac{D_{i} \tau_{i}\left(\beta_{i}^{2}-p_{i}^{2}\right)\left(h v-E_{g}\right)-E_{g}}{h \cup \kappa_{i} D_{i} \tau_{i}\left(\beta_{i}^{2}+k_{i}^{2}\right)\left(\beta_{i}^{2}-q_{i}^{2}\right)\left(\beta_{i}^{2}-p_{i}^{2}\right)} \beta_{i} I_{i}(x)\right] \\
& +\beta_{e i}\left[\frac{A_{i} e^{-p_{i} x}+B_{i} e^{p_{i} x}}{p_{i}^{2}+k_{i}^{2}}-\frac{\beta_{i} I_{i}(x)}{h v D_{i}\left(\beta_{i}^{2}+k_{i}^{2}\right)\left(\beta_{i}^{2}-p_{i}^{2}\right)}\right]
\end{aligned}
$$

\section{REFERENCES}

1. A. Mandelis, Photoacoustic and Thermal Wave Phenomena in Semiconductors (Elsevier Science, New York, 1987).

2. S. Y. Zhang and L. Chen, in Photoacoustic and Thermal Wave Phenomena in Semiconductors A. Mandelis, ed. (Elsevier Science, New York, 1987), p. 27.

3. J. Opsal and A. Rosencwaig, J. Appl. Phys. 53:4240 (1982).

4. Y. C. Shen and S. Y. Zhang, IEEE Trans. UFFC 39:227 (1992).

5. G. S. Kino and R. G. Stearn, Appl. Phys. Lett. 47:1048 (1985).

6. L. H. Wei, J. S. Xu, and S. Y. Zhang, IEEE Ultra. Symp. Proc. 501 (1986). 